\title{
Functional characterization and structural modeling of synthetic polyester-degrading hydrolases from Thermomonospora curvata
}

Ren Wei, Thorsten Oeser, Johannes Then, Nancy Kühn, Markus Barth, Juliane Schmidt and Wolfgang Zimmermann *

\begin{abstract}
Thermomonospora curvata is a thermophilic actinomycete phylogenetically related to Thermobifida fusca that produces extracellular hydrolases capable of degrading synthetic polyesters. Analysis of the genome of T. curvata DSM43183 revealed two genes coding for putative polyester hydrolases Tcur1278 and Tcur0390 sharing 61\% sequence identity with the T. fusca enzymes. Mature proteins of Tcur1278 and Tcur0390 were cloned and expressed in Escherichia coli TOP10. Tcur1278 and Tcur0390 exhibited an optimal reaction temperature against p-nitrophenyl butyrate at $60^{\circ} \mathrm{C}$ and $55^{\circ} \mathrm{C}$, respectively. The optimal pH for both enzymes was determined at pH 8.5. Tcur1278 retained more than $80 \%$ and Tcur0390 less than 10\% of their initial activity following incubation for 60 min at $55^{\circ} \mathrm{C}$. Tcur0390 showed a higher hydrolytic activity against poly( $(\varepsilon$-caprolactone) and polyethylene terephthalate (PET) nanoparticles compared to Tcur 1278 at reaction temperatures up to $50^{\circ} \mathrm{C}$. At $55^{\circ} \mathrm{C}$ and $60^{\circ} \mathrm{C}$, hydrolytic activity against PET nanoparticles was only detected with Tcur1278. In silico modeling of the polyester hydrolases and docking with a model substrate composed of two repeating units of PET revealed the typical fold of $\alpha / \beta$ serine hydrolases with an exposed catalytic triad. Molecular dynamics simulations confirmed the superior thermal stability of Tcur1278 considered as the main reason for its higher hydrolytic activity on PET.
\end{abstract}

Keywords: Polyester hydrolase; Synthetic polyester; Polyethylene terephthalate (PET); Thermomonospora curvata

\section{Introduction}

The widespread use of synthetic polyesters such as polyethylene terephthalate (PET) in industry and daily life has resulted in serious environmental pollution over the last decades. However, the recycling of PET by chemical methods performed under extreme temperature and $\mathrm{pH}$ conditions is an energy-consuming process (Paszun and Spychaj 1997). Recently, alternative processes using biocatalysis have been proposed for recycling and surface functionalization of PET (Müller et al. 2005; Zimmermann and Billig 2011). Microbial enzymes capable of degrading PET have been previously described from various fungal (Egmond and de Vlieg 2000; Alisch et al. 2004; Alisch-Mark et al. 2006; Nimchua et al. 2007; Ronkvist et al. 2009) and bacterial (Müller et al. 2005; Eberl et al. 2009; Herrero Acero et al. 2011; Ribitsch et al. 2012a;

\footnotetext{
* Correspondence: wolfgang.zimmermann@uni-leipzig.de Department of Microbiology and Bioprocess Technology, Institute of Biochemistry, University of Leipzig, Johannisallee 21-23, D-04103 Leipzig, Germany
}

Ribitsch et al. 2012b; Sulaiman et al. 2012; Kitadokoro et al. 2012; Chen et al. 2010; Oeser et al. 2010) sources. Enzymes with high PET-hydrolyzing activity are mostly extracellular proteins secreted by thermophilic microorganisms such as Thermomyces insolens (Ronkvist et al. 2009) and several Thermobifida species (Müller et al. 2005; Eberl et al. 2009; Herrero Acero et al. 2011; Ribitsch et al. 2012a; Ribitsch et al. 2012b; Kitadokoro et al. 2012; Chen et al. 2010; Oeser et al. 2010). The biodegradability of PET by these enzymes has been shown to strongly depend on the flexibility of polymer chains that is directly influenced by the hydrolysis reaction temperatures (Ronkvist et al. 2009; Wei et al. 2013).

Thermomonospora curvata DSM 43183, a facultative aerobic thermophilic actinomycete, has been isolated from composts containing plant materials (Henssen 1957; Henssen and Schnepf 1967; Chertkov et al. 2011). The optimal growth temperature of T. curvata is $50^{\circ} \mathrm{C}$ (Henssen and Schnepf 1967) at a wide range of $\mathrm{pH}$ from 7.5 to 11 (Chertkov et al. 2011). Weak growth of T. curvata has 
been also observed at higher temperatures up to $65^{\circ} \mathrm{C}$ (Henssen and Schnepf 1967). The phylogenetic analysis of $T$. curvata revealed a distant relationship to other thermophilic actinomycetes isolated from a similar habitat including Thermobifida fusca and Thermobifida $a l b a$, as indicated by a lower level of $16 \mathrm{~S}$ rRNA sequence similarity between $89 \%$ and 90\% (Henssen 1957; Zhang et al. 1998; Chertkov et al. 2011). Several of these bacteria have been shown to express extracellular enzymes with polyester-hydrolyzing activity (Kleeberg et al. 1998; Alisch et al. 2004; Herrero Acero et al. 2011; Thumarat et al. 2012; Ribitsch et al. 2012b).

In this study, we report the identification of two genes coding for the polyester hydrolases Tcur1278 and Tcur0390 by genome mining of T. curvata DSM43183 (Chertkov et al. 2011), the characterization of their catalytic properties and thermal stability, as well as the modeling and analysis of their three-dimensional structures.

\section{Materials and methods \\ Cloning, expression and purification of Tcur1278 and Tcur0390}

The genes encoding Tcur 1278 and Tcur0390 without the Gram-positive secretion signal peptides were selected from the annotated genome sequences of $T$. curvata DSM43183 (Chertkov et al. 2011). Synthetic gene constructs with adapted codon usage to $E$. coli (Geneart $\mathrm{GmbH}$, Regensburg, Germany) for Tcur1278 [EMBL: HG939554] and Tcur0390 [EMBL: HG939555] were applied for direct cloning into the pBAD TOPO expression vector (Invitrogen, Life Technologies, Carlsbad, USA). The recombinant expression of T. curvata hydrolases was carried out in One Shot E. coli TOP10 (Invitrogen) at room temperature for $14 \mathrm{~h}$ in lysogeny broth (LB) containing $0.2 \%(\mathrm{~m} / \mathrm{v})$ of L-arabinose as inducer as described previously (Oeser et al. 2010). Bacterial cells were harvested by centrifugation and resuspended in a lysis buffer containing $50 \mathrm{mM}$ phosphate $(\mathrm{pH} 8)$ and $300 \mathrm{mM} \mathrm{NaCl}$. After sonication, the soluble cell extracts were subjected to immobilized metal ion affinity chromatography (IMAC) using Ni-NTA columns (Qiagen, Hilden, Germany). The protein elutions containing the recombinant hydrolases were separated by SDS PAGE and analyzed by esterase activity-staining with 1-naphthyl acetate and Fast Red dye (Sztajer et al. 1992) as well as by staining with Coomassie Brilliant Blue.

\section{Determination of esterase activity}

Esterase activity was determined with p-nitrophenyl butyrate (pNPB) as a substrate in a microplate format (BioTek PowerWave XS, BioTek Instruments Inc., Winooski, USA) (Billig et al. 2010). To avoid the adsorption of proteins to the plastic vials, the dilution was carried out in the presence of $15 \%$ poly(ethylene glycol) ( $\mathrm{PEG}_{6000}$, Sigma-Aldrich
Co., St. Louis, USA) in Davies buffer (Davies 1959) between pH 6.5 and 9.5 or in $100 \mathrm{mM}$ Tris-HCl. One unit of esterase activity was defined as the amount of enzyme required to hydrolyze $1 \mu \mathrm{mol} \mathrm{pNPB}$ per min (Alisch et al. 2004). To investigate their thermal stability, $250 \mu \mathrm{g} / \mathrm{mL}$ of enzymes were incubated in $100 \mathrm{mM}$ Tris buffer $(\mathrm{pH}$ 8.5) at $50^{\circ} \mathrm{C}, 55^{\circ} \mathrm{C}$ and $60^{\circ} \mathrm{C}$ for up to $1 \mathrm{~h}$. Residual esterase activity against pNPB was determined at $25^{\circ} \mathrm{C}$ in triplicate. The Michaelis-Menten kinetic constants for the hydrolysis of pNPB by Tcur 1278 and Tcur0390 were determined at $25^{\circ} \mathrm{C}$ and $\mathrm{pH} 8.5$.

\section{Enzymatic hydrolysis of polyester nanoparticles}

The enzymatic hydrolysis of polyesters was analyzed by monitoring the change of turbidity of a polyester nanoparticle suspension at $600 \mathrm{~nm}$ (Wei et al. 2013). Poly(عcaprolactone) (PCL) and PET nanoparticles were prepared by a precipitation and solvent evaporation technique from amorphous PCL (Sigma-Aldrich Co., St. Louis, USA) and low-crystallinity PET film (Goodfellow $\mathrm{GmbH}$, Bad Nauheim, Germany) dissolved in acetone and 1,1,1,3,3,3-hexafluoro-2-propanol, respectively. The enzymatic hydrolysis of PCL was performed in a microplate format (BioTek PowerWave XS) at $49^{\circ} \mathrm{C}$ with $0.22 \mathrm{mg} / \mathrm{mL}$ of PCL nanoparticles in each well, whereas the enzymatic hydrolysis of PET was performed at $50^{\circ} \mathrm{C}$ to $60^{\circ} \mathrm{C}$ in cuvettes containing $0.25 \mathrm{mg} / \mathrm{mL}$ of PET nanoparticles immobilized in $0.9 \%$ agarose gel. The change of turbidity was monitored over an incubation period of $15 \mathrm{~min}$ at $1 \mathrm{~min}$ intervals for PCL hydrolysis, whereas PET hydrolysis was determined for $60 \mathrm{~min}$ at $5 \mathrm{~min}$ intervals. The initial degradation rates were defined as the square roots of turbidity decrease during the initial linear phase of the hydrolysis, and plotted as a function of enzyme concentration using a kinetic model (Eq. 1) modified from Wei et al. (2013)

$$
\frac{d(\sqrt{\tau})}{d t}=\frac{k_{\tau} K_{A}[E]}{1+K_{A}[E]}
$$

where $\tau$ is the turbidity of a nanoparticle suspension, $t$, the reaction time, $k_{v}$, the hydrolysis rate constant based on the turbidity change, $K_{A}$, the adsorption equilibrium constant, and $[E]$, the enzyme concentration.

\section{Homology modeling and molecular docking}

Homology modeling of $T$. curvata polyester hydrolases was carried out using the Phyre2 web server (Kelley and Sternberg 2009) based on the crystal structure of T. alba AHK 119 (Est119, PDB ID: 3VIS) (Kitadokoro et al. 2012). The sequence identity of $T$. curvata polyester hydrolases with the corresponding template structure is summarized in Table 1 and Additional file 1: Figure S1. 
Table 1 Sequence identity (in percent, upper right part) and the root-mean-square deviation (RMSD) of $C_{a}$ atoms (in $\AA$, lower left part) of $T$. curvata polyester hydrolases in comparison with the crystal structure of homologous T. alba Est119 (PDB ID: 3VIS)

\begin{tabular}{lccc}
\hline & \multicolumn{3}{c}{ Identity (\%) } \\
\cline { 2 - 4 } RMSD (Å) & Tcur1278 & Tcur0390 & T. alba Est119 \\
\hline Tcur1278 & & 82.2 & 61.7 \\
Tcur0390 & 0.11 & & 61.7 \\
T. alba Est119 & 0.85 & 0.83 & \\
\hline
\end{tabular}

The molecular docking program GOLD version 5.1 (Cambridge Crystallographic Data Centre, Cambridge, UK) (Jones et al. 1997) was used to study the substratebinding pocket of T. curvata polyester hydrolases. The polyester model substrate 2PET composed of 2 repeating units of PET (bis 2-hydroxyethyl terephthalate, BHET) was constructed with the software MOE (Chemical Computing Group, Montreal, Canada). The central ester bond of 2PET was constrained in the oxyanion hole composed of the main chain $\mathrm{NH}$ groups of amino acid residues F62 and M131 with the correct orientation to form a tetrahedral intermediate based on the catalytic mechanism of ester hydrolases (Jaeger et al. 1999). The other atoms of 2PET were allowed to be flexible for a conformation to be docked to the rigid protein structural model by a genetic algorithm (Jones et al. 1997). Based on the default scoring function of GOLD, the topranked productive docking conformations in accordance with the catalytic mechanism of ester hydrolases (Jaeger et al. 1999) were selected for the illustrations generated by the MOE software.

\section{Molecular dynamics simulations}

The molecular dynamics (MD) simulations were carried out using GROMACS 4.6 (Groningen University, The Netherlands) (Hess et al. 2008) in the Amber99SB force field (Hornak et al. 2006) in explicit solvent. Protein structural models of both $T$. curvata polyester hydrolases were centered in a cube with a distance of $\geq 1.0 \mathrm{~nm}$ from each edge as the starting structures. The steepest descent method was applied to perform energy minimization until a maximum force $\left(\mathrm{F}_{\max }\right)$ of less than $1000 \mathrm{~kJ} / \mathrm{mol} / \mathrm{nm}$ was reached. The system was equilibrated for 100 ps by a position-restrained simulation at the desired temperatures in the isothermal-isobaric (NPT) ensemble. The isotropic pressure coupling using the Berendsen algorithm was applied with a reference pressure of 1.0 bar (Berendsen et al. 1984). For each protein structure, three independent simulations were performed under equilibration conditions for $50 \mathrm{~ns}$ in 2 fs steps at $298 \mathrm{~K}\left(25^{\circ} \mathrm{C}\right)$ and $353 \mathrm{~K}\left(80^{\circ} \mathrm{C}\right)$, respectively. To analyze the thermal stability of the polyester hydrolases, the time course of the root-mean-square deviation (RMSD) of backbone structures and the rootmean-square fluctuation (RMSF) of $\mathrm{C}_{\alpha}$ atoms of each amino acid residue over the complete 50 ns simulation were calculated using GROMACS 4.6 (Hess et al. 2008).

\section{Results \\ Cloning, expression and purification of Tcur1278 and Tcur0390}

Synthetic genes encoding Tcur1278 and Tcur0390 were amplified in the pBAD-TOPO expression vector (Invitrogen) for recombinant expression in One Shot E. coli TOP10 (Invitrogen). Following an expression period of $14 \mathrm{~h}$ at $25^{\circ} \mathrm{C}$ and the subsequent IMAC purification, $2.5 \mathrm{mg}$ of Tcur1278 and $2.9 \mathrm{mg}$ of Tcur0390 were obtained from a $500 \mathrm{~mL}$ culture with a specific activity of 3.0 $\mathrm{U} / \mathrm{mg}$ and $17.9 \mathrm{U} / \mathrm{mg}$ against pNPB, respectively. By SDS PAGE analysis, both $T$. curvata hydrolases were obtained as single bands with esterase activity against 1-naphthyl acetate, corresponding to an apparent molecular mass of approximately $35 \mathrm{kDa}$ (Additional file 1: Figure S2).

\section{Effect of $\mathrm{pH}$ and temperature on the hydrolytic activity of} Tcur1278 and Tcur0390

The effect of $\mathrm{pH}$ on the hydrolytic activity of Tcur1278 and Tcur0390 was investigated against $\mathrm{pNPB}$ in a $\mathrm{pH}$ range from 6.5 to 9.5 (Figure 1A). Both enzymes displayed an optimal $\mathrm{pH}$ at $\mathrm{pH} 8.5$ and still retained more than $60 \%$ of their maximum activity at $\mathrm{pH} 9.5$.

The effect of temperature on the hydrolytic activity of both enzymes was assayed against pNPB in a temperature range from $30^{\circ} \mathrm{C}$ to $70^{\circ} \mathrm{C}$ (Figure $1 \mathrm{~B}$ ). Tcur1278 and Tcur0390 showed an optimal temperature at $60^{\circ} \mathrm{C}$ and $55^{\circ} \mathrm{C}$, respectively.

\section{Thermal stability of Tcur1278 and Tcur0390}

The stability of Tcur 1278 and Tcur 0390 at $50^{\circ} \mathrm{C}, 55^{\circ} \mathrm{C}$ and $60^{\circ} \mathrm{C}$ was investigated at $\mathrm{pH} 8.5$ over a period of $60 \mathrm{~min}$ by monitoring the residual activities against pNPB (Figure 2A-B). Tcur1278 showed a higher thermal stability compared to Tcur0390 retaining more than $80 \%$ of its initial activity following incubation for $60 \mathrm{~min}$ at $50^{\circ} \mathrm{C}$ and $55^{\circ} \mathrm{C}$. At $60^{\circ} \mathrm{C}$, approximately $65 \%$ loss of its initial activity was detected following incubation for $10 \mathrm{~min}$. In contrast, Tcur0390 showed a residual activity of only $40 \%$ following incubation for $60 \mathrm{~min}$ at $50^{\circ} \mathrm{C}$ and of $15 \%$ following incubation for $10 \mathrm{~min}$ at $55^{\circ} \mathrm{C}$ and $60^{\circ} \mathrm{C}$.

\section{Kinetic analysis of the hydrolysis of pNPB by Tcur1278 and Tcur0390}

Based on the Michaelis-Menten kinetic model, Tcur0390 revealed an almost 6-fold higher $k_{\text {cat }}$ and no significantly lower $K_{\mathrm{m}}$ than Tcur1278 for pNPB hydrolysis indicating a higher hydrolytic activity of Tcur0390 against the soluble pNPB compared to Tcur1278 (Table 2). 

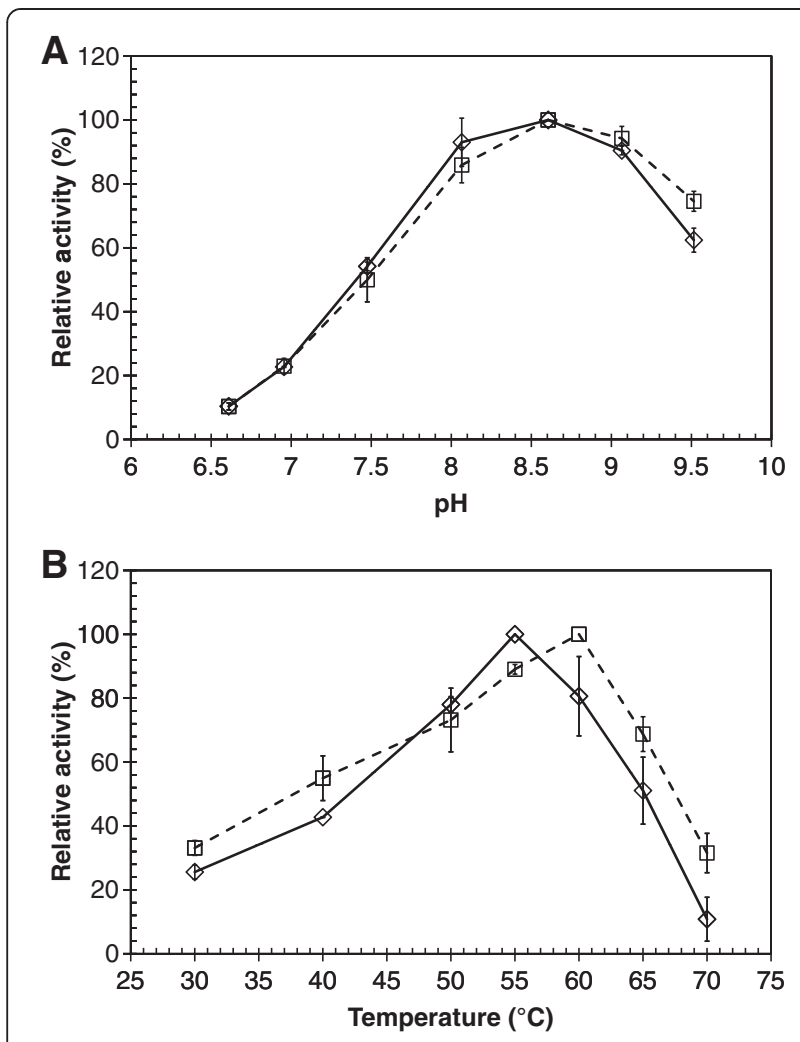

Figure 1 Effects of $\mathrm{pH}$ and temperature on the hydrolytic activity of Tcur1278 and Tcur0390. Activities of Tcur 1278 and Tcur0390 against pNPB at different $(\mathbf{A}) \mathrm{pH}$ and $\mathbf{( B )}$ reaction temperature conditions are shown as broken and solid lines, respectively. Error bars indicate the standard deviation of three determinations.

Kinetic analysis of the hydrolysis of PCL nanoparticles by Tcur1278 and Tcur0390

A PCL nanoparticle suspension with a concentration of $0.22 \mathrm{mg} / \mathrm{mL}$ was completely hydrolyzed following incubation for $15 \mathrm{~min}$ at $49^{\circ} \mathrm{C}$ with $20 \mu \mathrm{g} / \mathrm{mL}$ of Tcur0390 or $30 \mu \mathrm{g} / \mathrm{mL}$ of Tcur1278 (data not shown). The kinetic analysis of the hydrolysis of PCL nanoparticles was therefore performed at enzyme concentrations up to $20 \mu \mathrm{g} / \mathrm{mL}$ and $30 \mu \mathrm{g} / \mathrm{mL}$ for Tcur0390 and Tcur1278, respectively. The hydrolysis rates of PCL nanoparticles calculated from the square roots of turbidity decrease are shown as a function of enzyme concentration (Figure 3A-B). By fitting the experimental data to Eq. (1), the kinetic constants for the PCL nanoparticle hydrolysis by the two enzymes were determined (Table 3). Compared to Tcur1278, Tcur0390 showed a 2.3-fold higher adsorption equilibrium constant $\left(K_{A}\right)$ and no significantly higher hydrolysis rate constant $\left(k_{\tau}\right)$.

\section{Kinetic analysis of the hydrolysis of PET nanoparticles by} Tcur1278 and Tcur0390

The enzymatic hydrolysis of PET nanoparticles by Tcur1278 and Tcur0390 was investigated at $\mathrm{pH} 8.5$ and temperatures of $50^{\circ} \mathrm{C}, 55^{\circ} \mathrm{C}$ and $60^{\circ} \mathrm{C}$ (Figure $3 \mathrm{C}-\mathrm{F}$ ). Due to the lower
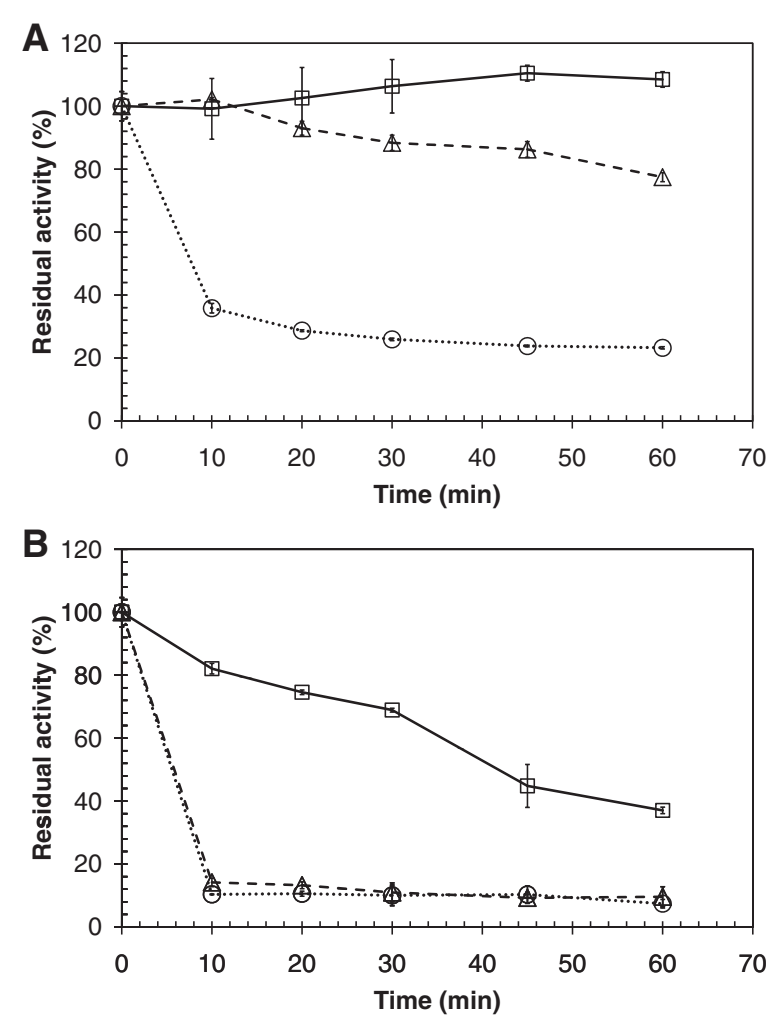

Figure 2 Thermal stability performance of Tcur1278 and Tcur0390. The residual hydrolytic activity was determined with (A) Tcur1278 and (B) Tcur0390 against pNPB over a period of $1 \mathrm{~h}$ at $50^{\circ} \mathrm{C}$ (solid line), $55^{\circ} \mathrm{C}$ (broken line) and $60^{\circ} \mathrm{C}$ (dotted line). Error bars indicate the standard deviation of three determinations.

thermal stability of Tcur0390 (Figure 2B), a hydrolytic activity at $55^{\circ} \mathrm{C}$ and $60^{\circ} \mathrm{C}$ was not detected. At $50^{\circ} \mathrm{C}$, a maximum hydrolysis rate $(d(\sqrt{\tau}) / d t)$ of $3.3 \times 10^{-3} \mathrm{~min}^{-1}$ and $5.9 \times 10^{-3} \mathrm{~min}^{-1}$ was determined with $80 \mu \mathrm{g} / \mathrm{mL}$ of Tcur1278 and $20 \mu \mathrm{g} / \mathrm{mL}$ of Tcur0390, respectively. With $50 \mu \mathrm{g} / \mathrm{mL}$ of Tcur1278, the hydrolysis rate was increased 1.8 -fold at $55^{\circ} \mathrm{C}$ and 2.6 -fold at $60^{\circ} \mathrm{C}$. Higher enzyme concentrations exceeding the amount required for the maximum reaction rate resulted in lower hydrolysis rates. This effect has also been observed in the hydrolysis of PET nanoparticles by TfCut2, a polyester hydrolase from T. fusca, and has been attributed to the adsorption of catalytically inactive enzyme in excess to the monolayer coverage of the PET surface (Wei et al. 2013).

Table 3 summarizes the kinetic constants for the enzymatic PET nanoparticle hydrolysis by fitting the

Table 2 Kinetic parameters for pNPB hydrolysis by Tcur1278 and Tcur0390 at $25^{\circ} \mathrm{C}$ and $\mathrm{pH} 8.5$

\begin{tabular}{lcc}
\hline & Tcur1278 & Tcur0390 \\
\hline $\boldsymbol{K}_{\mathrm{m}}(\boldsymbol{\mu M})$ & $88.8 \pm 12.8$ & $83.1 \pm 11.1$ \\
$\boldsymbol{k}_{\text {cat }}(\mathbf{1} / \mathbf{s})$ & $2.3 \pm 0.1$ & $12.4 \pm 0.4$ \\
\hline
\end{tabular}



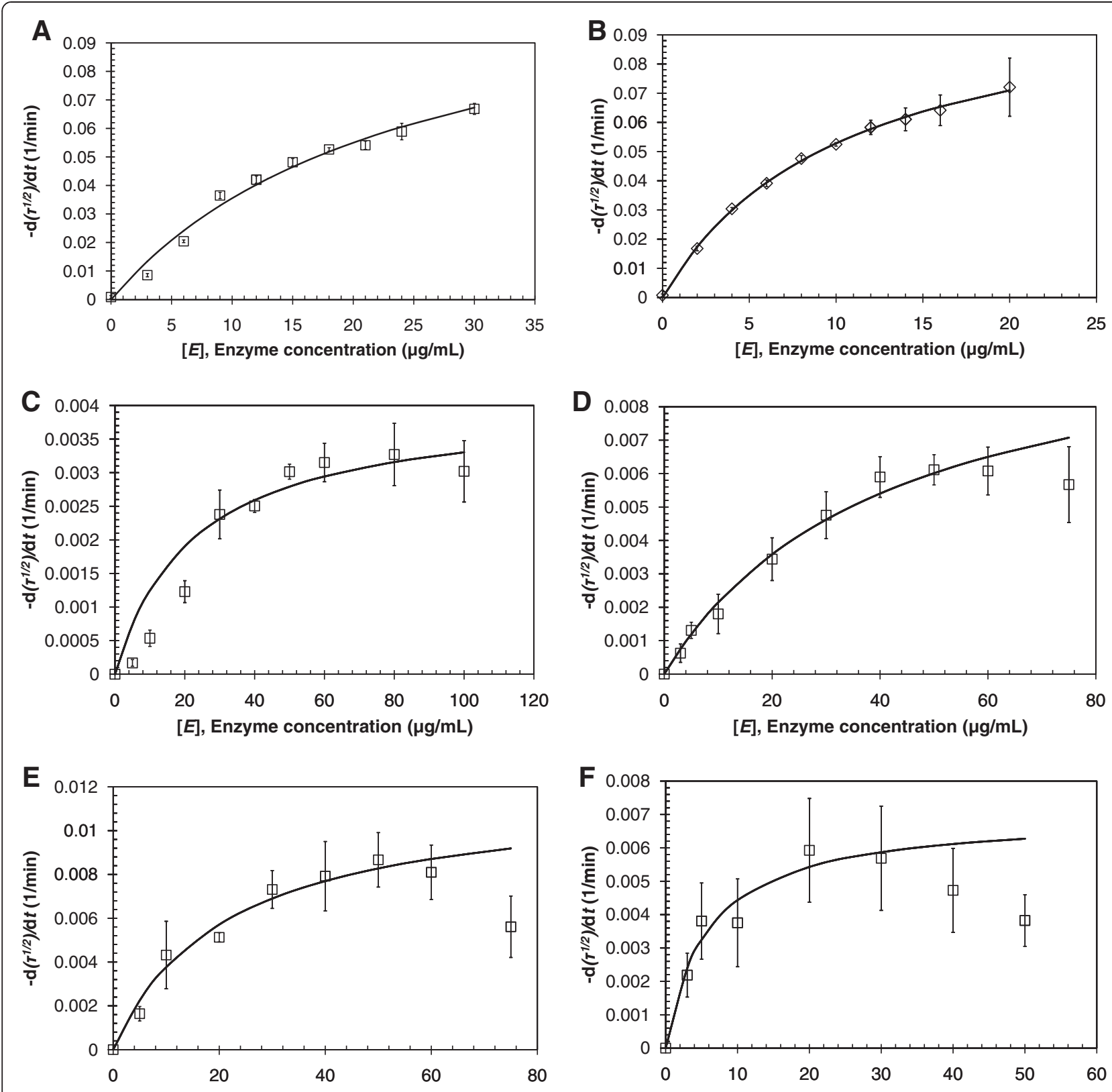

$[E]$, Enzyme concentration $(\mu \mathrm{g} / \mathrm{mL})$

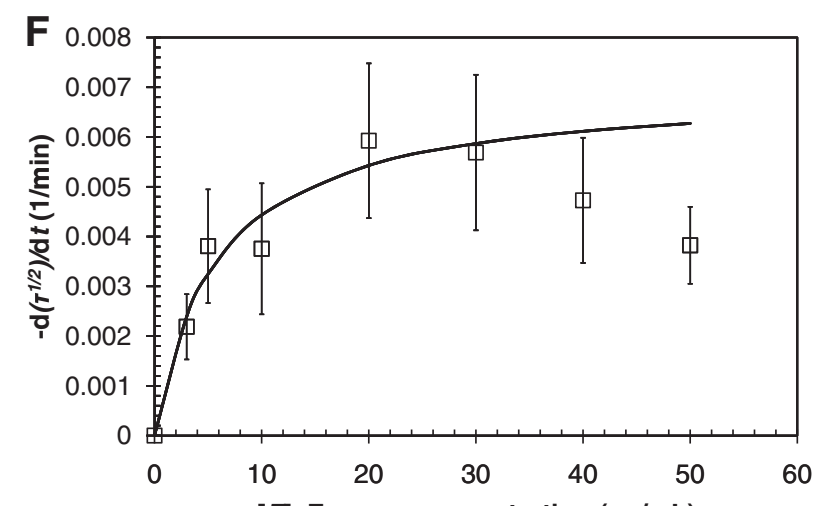

Figure 3 Decomposition of polyester nanoparticles by $T$. curvata hydrolases. PCL hydrolysis by (A) Tcur 1278 and (B) Tcur0390 at $49^{\circ} \mathrm{C} ;$ PET hydrolysis by Tcur 1278 at (C) $50^{\circ} \mathrm{C}$, (D) $55^{\circ} \mathrm{C}$ and (E) $60^{\circ} \mathrm{C}$, and by Tcur0390 at (F) $50^{\circ} \mathrm{C}$. The initial rates of the square roots of turbidity decrease are plotted as a function of enzyme concentration (squares and diamonds). Error bars represent the standard deviation of duplicate determinations. Fitted data (solid lines) according to Eq. (1) are also shown.

experimental data to Eq. (1). Compared to Tcur1278, a 1.7-fold higher $k_{\tau}$ and a 3.9-fold higher $K_{A}$ were obtained with Tcur0390 at $50^{\circ} \mathrm{C}$. With Tcur 1278 , the highest values of both kinetic constants were determined at $60^{\circ} \mathrm{C}$.

\section{In silico modeling of Tcur1278 and Tcur0390}

Structural models of Tcur1278 and Tcur0390 were generated on the basis of the crystal structure of Est119 from T. alba AHK119 (PDB ID: 3VIS) (Kitadokoro et al.
2012). Homology models of T. curvata polyester hydrolases revealed a typical $\alpha / \beta$ hydrolase fold (Ollis et al. 1992; Carr and Ollis 2009) with low RMSD values of $C_{\alpha}$ atomic coordinates of less than $1 \AA$ in comparison with the template crystal structure (Table 1).

Similar to TfCut2 from T. fusca KW3 (Wei 2011; Herrero Acero et al. 2011), the catalytic triad of T. curvata polyester hydrolases formed by S130, D176 and H208 was found to be exposed to the solvent 
Table 3 Kinetic parameters for polyester nanoparticle hydrolysis by Tcur1278 and Tcur0390 at $49^{\circ} \mathrm{C}$ to $60^{\circ} \mathrm{C}$ and pH 8.5

\begin{tabular}{|c|c|c|c|c|c|}
\hline \multirow{2}{*}{\multicolumn{2}{|c|}{$\begin{array}{c}\text { Polyester nanoparticles } \\
\text { Temperature }\left({ }^{\circ} \mathrm{C}\right)\end{array}$}} & \multirow{3}{*}{$\begin{array}{c}\text { PCL } \\
\mathbf{4 9} \\
41.1 \pm 4.5\end{array}$} & \multicolumn{3}{|c|}{ PET } \\
\hline & & & \multirow{2}{*}{$\begin{array}{c}\mathbf{5 0} \\
44.4 \pm 8.6\end{array}$} & \multirow{2}{*}{$\frac{55}{24.3 \pm 5.3}$} & \multirow{2}{*}{$\frac{60}{46.9 \pm 12}$} \\
\hline Tour1278 & $K_{\mathrm{A}}(\mathrm{mL} / \mathrm{mg})$ & & & & \\
\hline reurizio & $k_{\tau}\left(10^{-3} / \mathrm{min}\right)$ & $122.2 \pm 11.9$ & $4.1 \pm 0.5$ & $11.0 \pm 1.2$ & $11.8 \pm 1.2$ \\
\hline \multirow{2}{*}{ Tcur0390 } & $K_{\mathrm{A}}(\mathrm{mL} / \mathrm{mg})$ & $96.0 \pm 9.8$ & $172.7 \pm 30.7$ & n. d. & n. d. \\
\hline & $k_{\tau}\left(10^{-3} / \mathrm{min}\right)$ & $108.3 \pm 5.7$ & $7.0 \pm 0.8$ & n. $d$. & n. d. \\
\hline
\end{tabular}

n. d. $=$ not detectable.

(Figure 4A-B). By docking of the 2PET model substrate, the substrate-binding pocket could be identified as a large groove on the surface of Tcur1278 and Tcur0390 (Figure 4C-F). The negative charge buried in this major groove was contributed by the deprotonated S130 (Figure 4C-D). As shown in Figure 4C-F, Tcur1278 and Tcur0390 displayed similar surface properties in the vicinity of the active site with extended hydrophobic regions around the substrate-binding groove.

Molecular dynamics simulations of Tcur1278 and Tcur0390 The overall $C_{\alpha}$ RMSD values for $T$. curvata polyester hydrolases obtained by MD simulations at $298 \mathrm{~K}$ and $353 \mathrm{~K}$ are shown as a function of simulation time (Figure 5A-B). In all simulations, the RMSD values for both proteins stabilized rapidly within 0.02 ns. At $298 \mathrm{~K}$, the RMSD for Tcur 1278 showed values below $0.1 \mathrm{~nm}$, slightly lower than the corresponding values for Tcur0390. At $353 \mathrm{~K}$, the RMSD values for Tcur0390 fluctuated more strongly compared to Tcur1278. This effect was most pronounced after 15 ns simulation time. The RMSD values for Tcur0390 were almost doubled at $353 \mathrm{~K}$ compared to those obtained at $298 \mathrm{~K}$. In contrast, Tcur1278 exhibited only a slight increase in backbone structure deviations at $353 \mathrm{~K}$ further confirming its superior thermal stability properties.

The corresponding RMSF plots revealed the flexibility profiles for the complete protein sequence (Figure 5C-D). Both the enzymes displayed a flexible $\mathrm{N}$-terminus with the highest deviation of $\mathrm{C}_{\alpha}$ atoms contributed mainly by a short helical part of the molecule (12-17) embedded in loop structures $(2-11,18-23)$. The high RMSF observed in this part of the protein also affected the neighboring betasheet structure (24-30) and furthermore the whole enzyme. Compared to Tcur1278, Tcur0390 showed generally a larger difference in the flexibility profiles obtained by the MD simulations performed at temperatures from $298 \mathrm{~K}$ to $353 \mathrm{~K}$. A significant increase of the RMSF values at higher temperatures was observed in the neighborhood of D176 (172-180) with both enzymes. This resulted also in a higher flexibility of $\mathrm{H} 208$ at $353 \mathrm{~K}$ due to its interaction with D176 according to the catalytic mechanism of ester hydrolases (Jaeger et al. 1999). As a consequence, the distance between the catalytic H208 and S130 (H-S) increased from $0.3 \mathrm{~nm}$ to $0.5 \mathrm{~nm}$ after $34 \mathrm{~ns}$ and $28 \mathrm{~ns}$ of the MD simulation at $353 \mathrm{~K}$ with Tcur1278 and Tcur0390, respectively (Figure 5E-F). Compared to the RMSD plot shown in Figure 5A that suggested a relatively stable backbone structure of Tcur1278 during the complete 50 ns simulation at $353 \mathrm{~K}$, the permanent change of the H-S distance occurred at an earlier stage prior to the denaturation of its other temperature-labile parts.

\section{Discussion}

Bacterial polyester hydrolases have been previously described mainly from thermophilic Thermobifida species (Kleeberg et al. 1998; Alisch et al. 2004; Herrero Acero et al. 2011; Thumarat et al. 2012; Ribitsch et al. 2012b; Oeser et al. 2010). T. curvata is a phylogenetically related actinomycete that has been isolated from a similar habitat (Henssen 1957; Zhang et al. 1998; Chertkov et al. 2011). By genome mining of T. curvata DSM 43183 (Chertkov et al. 2011), we identified two genes encoding the proteins Tcur1278 and Tcur0390 with sequences similar to TfCut2 from T. fusca KW3 (Wei 2011; Herrero Acero et al. 2011). As shown in the protein sequence alignment (Simossis and Heringa 2005), Tcur1278 and Tcur0390 share a sequence identity of about $82 \%$ and both enzymes show a sequence identity of about $61 \%$ with TfCut2 (Additional file 1: Figure S1).

Codon-optimized genes of Tcur1278 and Tcur0390 were synthesized for cloning and recombinant expression in E. coli. When the pET-20b(+) vector (Novagen) was used for the recombinant expression of the complete proteins of Tcur1278 and Tcur0390 in E. coli BL21(DE3), no active proteins could be detected suggesting an interference of the original Gram-positive signal peptides with the recombinant system. With the pBAD expression vector and $E$. coli TOP10 (Invitrogen) for shorter mature proteins, both $T$. curvata polyester hydrolases could be expressed as active enzymes fused with a C-terminal His-tag and purified by affinity chromatography (Additional file 1: Figure S2).

Similar to homologous polyester hydrolases from T. fusca (Chen et al. 2008; Wei 2011; Herrero Acero et al. 2011), Tcur1278 and Tcur0390 showed their highest activity against pNPB between $\mathrm{pH} 8$ and $\mathrm{pH} 9$ in a temperature range from $50^{\circ} \mathrm{C}$ to $60^{\circ} \mathrm{C}$ (Figure 1). Compared to Tcur1278, Tcur0390 revealed a significantly higher 


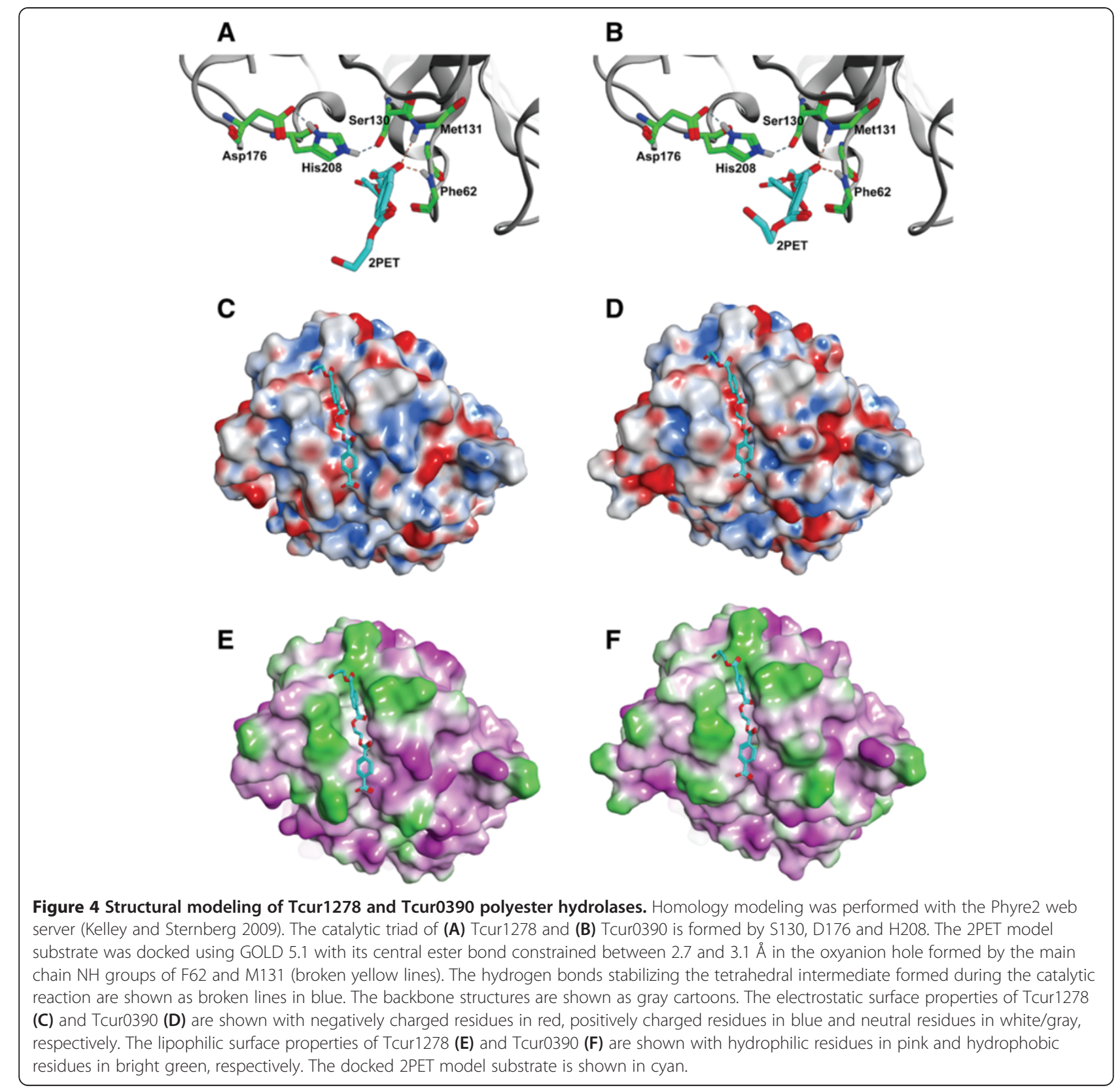

hydrolytic activity against both soluble (pNPB) and insoluble substrates (polyester nanoparticles) at reaction temperatures up to $50^{\circ} \mathrm{C}$ (Figure 3, Tables 2 and 3). This higher hydrolytic activity could be attributed to the stronger substrate affinity of Tcur0390 (Table 3). Molecular docking experiments with the model substrate 2PET to the structural models of T. curvata polyester hydrolases confirmed the presence of extended hydrophobic regions in close vicinity to the catalytic triad (Figure 4E-F). The hydrophobic character of the regions near the substrate-binding groove may facilitate the binding of hydrophobic polymeric substrates. Compared to Tcur1278, the hydrophobic properties were more pronounced in Tcur0390 and may account for its observed higher substrate affinity (Figure 4E-F). This result is confirming earlier observations that more hydrophobic and less charged amino acid residues clustered in the neighborhood of the substrate-binding groove of Thc_Cut1 compared to Thc_Cut2 and a concomitantly higher hydrolytic activity of the former isoenzyme from $T$. cellulosilytica (Herrero Acero et al. 2011; Herrero Acero et al. 2013).

The optimal temperatures for pNPB hydrolysis by Tcur 1278 and Tcur 0390 were $60^{\circ} \mathrm{C}$ and $55^{\circ} \mathrm{C}$, respectively (Figure 1B). However, both enzymes showed poor thermal stability at their optimal temperature, as indicated by an 


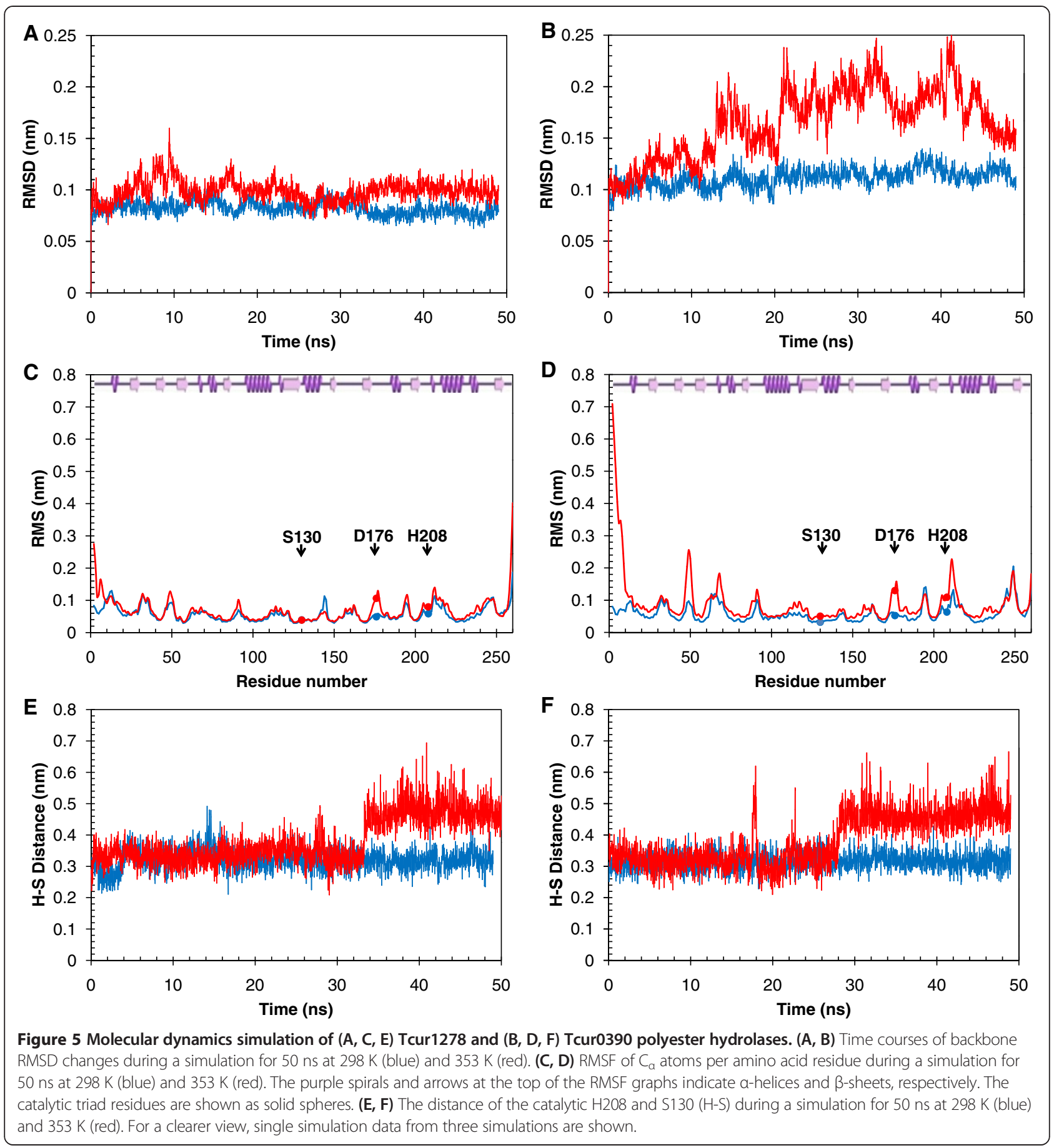

irreversible loss of more than $65 \%$ of their initial activities following incubation for $10 \mathrm{~min}$ (Figure 2A-B). Tcur1278 maintained its maximum activity against PET nanoparticles for approximately $15 \mathrm{~min}$ at $60^{\circ} \mathrm{C}$ (data not shown). This suggests that the thermal stability of Tcur1278 was improved in the presence of the insoluble polymeric substrate. In contrast, a significant improvement of the thermal stability was not detected with Tcur0390 in the presence of PET nanoparticles. The backbone RMSD plots obtained by MD simulations also indicated a more rigid structure of Tcur1278 thus verifying its superior thermal stability compared to Tcur0390 (Figure 5A-B). The RMSF profiles that describe the deviation of $C_{\alpha}$ atoms of single amino acid residues from the averaged position over the simulation period showed highly flexible regions clustered in the neighborhood of the catalytic residues H208 and D176 (Figure 5C-D). These regions may enable some induced fit motions necessary for the catalytic reaction at 
the active site. A comparison of the backbone RMSD plots (Figure 5A) and the H-S distance of Tcur1278 (Figure 5E) over the complete MD simulation period indicated that the exposed flexible catalytic triad was also prone to local unfolding prior to the denaturation of the overall structure. By contrast, the permanent increase of the H-S distance in Tcur0390 was accompanied by the unfolding of the overall structure and occurred at an earlier stage of MD simulations compared to Tcur1278 (Figure 5B, F).

A reaction temperature close to the glass transition temperature of PET at approximately $75^{\circ} \mathrm{C}$ (Alves et al. 2002) is required for an optimal performance of the enzymatic hydrolysis due to the restricted mobility of polymer chains at temperatures below (Marten et al. 2003, 2005; Herzog et al. 2006; Ronkvist et al. 2009; Wei et al. 2013). The thermal stability of Tcur1278 needs therefore be further improved for an efficient degradation of PET. Protein engineering in regions near the catalytic triad as well as at the flexible $\mathrm{N}$-terminus could be a useful approach for further optimizations to overcome the limited thermal stability of these polyester hydrolases.

In summary, the polyester hydrolases Tcur1278 and Tcur0390 from $T$. curvata have been shown to exhibit catalytic and structural features similar to enzymes from T. fusca and T. cellulosilytica. The comparison of the catalytic characteristics of Tcur1278 and Tcur0390 revealed a correlation between their hydrolytic activity and their surface properties in the vicinity of the catalytic triad. However, a comparison of the thermal stability of the two enzymes provided evidence that their ability to hydrolyze PET is predominately limited by their stability at higher reaction temperatures.

\section{Additional file}

\section{Additional file 1: Figure S1. Alignment of the mature protein} sequences of Tcur1278, Tcur0390, TfCut2 and Est119 polyester hydrolases. The regions of similarity of individual amino acid residues are indicated with colors from blue, unconserved to red, conserved. The multiple sequence alignment was performed with the PRALINE web server (Simossis and Heringa 2005). Figure S2. SDS PAGE analysis of Tcur0390 (lanes 1-2) and Tcur1278 (lanes 3-4). $10 \mu \mathrm{g}$ of crude cell lysate $(1,3)$ and eluate obtained after IMAC purification $(2,4)$ were loaded in each lane; protein size markers (M). The gel was first stained with Fast Red dye for esterase activity against 1-naphthyl acetate (purple bands) followed by staining with Coomassie Brilliant Blue (blue bands).

\section{Competing interests}

The authors declare that they have no competing interests.

\section{Authors' contributions}

RW and NK carried out the recombinant cloning of genes coding for the polyester hydrolases. MB and JS participated in the expression and purification of the recombinant enzymes. TO and RW carried out the biochemical characterization of the polyester hydrolases. JT performed the molecular dynamics simulations. RW and TO analyzed the experimental and simulation data and prepared the manuscript. WZ conceived the study and contributed to manuscript writing. All authors read and approved the final manuscript.

\section{Acknowledgements}

Dr. René Meier, Institute of Biochemistry, University of Leipzig is acknowledged for his assistance in the MD simulations. This work was supported by the Deutsche Bundesstiftung Umwelt (AZ 13267; AZ 2012/202).

Received: 20 April 2014 Accepted: 27 April 2014

Published online: 03 June 2014

\section{References}

Alisch-Mark M, Herrmann A, Zimmermann W (2006) Increase of the hydrophilicity of polyethylene terephthalate fibres by hydrolases from Thermomonospora fusca and Fusarium solani f. sp. pisi. Biotechnol Lett 28(10):681-685. doi:10.1007/s10529-006-9041-7

Alisch M, Feuerhack A, Müller H, Mensak B, Andreaus J, Zimmermann W (2004) Biocatalytic modification of polyethylene terephthalate fibres by esterases from actinomycete isolates. Biocatal Biotransform 22(5-6):347-351. doi:10.1080/10242420400025877

Alves NM, Mano JF, Balaguer E, Meseguer Duenas JM, Gomez Ribelles JL (2002) Glass transition and structural relaxation in semi-crystalline poly(ethylene terephthalate): a DSC study. Polymer 43(15):4111-4122. doi:10.1016/S00323861(02)00236-7

Berendsen HJC, Postma JPM, van Gunsteren WF, DiNola A, Haak JR (1984) Molecular dynamics with coupling to an external bath. J Chem Phys 81 (8):3684-3690

Billig S, Oeser T, Birkemeyer C, Zimmermann W (2010) Hydrolysis of cyclic poly (ethylene terephthalate) trimers by a carboxylesterase from Thermobifida fusca KW3. Appl Microbiol Biotechnol 87(5):1753-1764. doi:10.1007/s00253010-2635-y

Carr PD, Ollis DL (2009) Alpha/beta hydrolase fold: an update. Protein Pept Lett 16(10):1137-1148. doi:10.2174/092986609789071298

Chen S, Su L, Billig S, Zimmermann W, Chen J, Wu J (2010) Biochemical characterization of the cutinases from Thermobifida fusca. J Mol Catal B-Enzym 63(3-4):121-127. doi:10.1016/j.molcatb.2010.01.001

Chen S, Tong X, Woodard RW, Du G, Wu J, Chen J (2008) Identification and characterization of bacterial cutinase. J Biol Chem 283(38):25854-25862. doi:10.1074/jbc.M800848200

Chertkov O, Sikorski J, Nolan M, Lapidus A, Lucas S, Del Rio TG, Tice H, Cheng JF, Goodwin L, Pitluck S, Liolios K, Ivanova N, Mavromatis K, Mikhailova N, Ovchinnikova G, Pati A, Chen A, Palaniappan K, Djao OD, Land M, Hauser L, Chang YJ, Jeffries CD, Brettin T, Han C, Detter JC, Rohde M, Goker M, Woyke T, Bristow J, Eisen JA, Markowitz V, Hugenholtz P, Klenk HP, Kyrpides NC (2011) Complete genome sequence of Thermomonospora curvata type strain (B9). Stand Genomic Sci 4(1):13-22. doi:10.4056/sigs.1453580

Davies MT (1959) A universal buffer solution for use in ultra-violet spectrophotometry. Analyst 84:248-251. doi:10.1039/AN9598400248

Eberl A, Heumann S, Bruckner T, Araujo R, Cavaco-Paulo A, Kaufmann F, Kroutil W, Guebitz GM (2009) Enzymatic surface hydrolysis of poly(ethylene terephthalate) and bis(benzoyloxyethyl) terephthalate by lipase and cutinase in the presence of surface active molecules. J Biotechnol 143(3):207-212. doi:10.1016/j. jbiotec.2009.07.008

Egmond MR, de Vlieg J (2000) Fusarium solani pisi cutinase. Biochimie 82(11):1015-1021. doi:10.1016/S0300-9084(00)01183-4

Henssen A (1957) Beitraege zur Morphologie und Systematik der thermophilen Actinomyceten. Arch Mikrobiol 26(4):373-414. doi:10.1007/bf00407588

Henssen A, Schnepf E (1967) Zur Kenntnis thermophiler Actinomyceten. Arch Mikrobiol 57(3):214-231. doi:10.1007/bf00405948

Herrero Acero E, Ribitsch D, Dellacher A, Zitzenbacher S, Marold A, Steinkellner G, Gruber K, Schwab H, Guebitz GM (2013) Surface engineering of a cutinase from Thermobifida cellulosilytica for improved polyester hydrolysis. Biotechnol Bioeng 110(10):2581-2590. doi:10.1002/bit.24930

Herrero Acero E, Ribitsch D, Steinkellner G, Gruber K, Greimel K, Eiteljoerg I, Trotscha E, Wei R, Zimmermann W, Zinn M, Cavaco-Paulo A, Freddi G, Schwab H, Guebitz G (2011) Enzymatic surface hydrolysis of PET: Effect of structural diversity on kinetic properties of cutinases from Thermobifida. Macromolecules 44(12):4632-4640. doi:10.1021/ma200949p

Herzog K, Müller RJ, Deckwer WD (2006) Mechanism and kinetics of the enzymatic hydrolysis of polyester nanoparticles by lipases. Polym Degrad Stab 91(10):2486-2498. doi:10.1016/j.polymdegradstab.2006.03.005

Hess B, Kutzner C, van der Spoel D, Lindahl E (2008) GROMACS 4: Algorithms for highly efficient, load-balanced, and scalable molecular simulation. J Chem Theory Comput 4(3):435-447. doi:10.1021/ct700301q 
Hornak V, Abel R, Okur A, Strockbine B, Roitberg A, Simmerling C (2006) Comparison of multiple Amber force fields and development of improved protein backbone parameters. Proteins 65(3):712-725. doi:10.1002/prot.21123

Jaeger KE, Dijkstra BW, Reetz MT (1999) Bacterial biocatalysts: molecular biology, three-dimensional structures, and biotechnological applications of lipases. Annu Rev Microbiol 53:315-351. doi:10.1146/annurev.micro.53.1.315

Jones G, Willett P, Glen RC, Leach AR, Taylor R (1997) Development and validation of a genetic algorithm for flexible docking. J Mol Biol 267(3):727-748. doi:10.1006/ jmbi.1996.0897

Kelley LA, Sternberg MJ (2009) Protein structure prediction on the Web: a case study using the Phyre server. Nat Protoc 4(3):363-371. doi:10.1038/nprot.2009.2

Kitadokoro K, Thumarat U, Nakamura R, Nishimura K, Karatani H, Suzuki H, Kawai F (2012) Crystal structure of cutinase Est119 from Thermobifida alba AHK119 that can degrade modified polyethylene terephthalate at $1.76 \AA$ resolution. Polym Degrad Stab 97(5):771-775. doi:10.1016/j.polymdegradstab.2012.02.003

Kleeberg I, Hetz C, Kroppenstedt RM, Muller RJ, Deckwer WD (1998) Biodegradation of aliphatic-aromatic copolyesters by Thermomonospora fusca and other thermophilic compost isolates. Appl Environ Microbiol 64(5):1731-1735

Müller R-J, Schrader H, Profe J, Dresler K, Deckwer W-D (2005) Enzymatic degradation of poly(ethylene terephthalate): rapid hydrolyse using a hydrolase from $T$. fusca. Macromol Rapid Commun 26(17):1400-1405. doi:10.1002/marc.200500410

Marten E, Müller R-J, Deckwer W-D (2003) Studies on the enzymatic hydrolysis of polyesters I. Low molecular mass model esters and aliphatic polyesters. Polym Degrad Stab 80(3):485-501. doi:10.1016/S0141-3910(03)00032-6

Marten E, Müller R-J, Deckwer W-D (2005) Studies on the enzymatic hydrolysis of polyesters. II Aliphatic-aromatic copolyesters. Polym Degrad Stab 88(3):371-381. doi:10.1016/j.polymdegradstab.2004.12.001

Nimchua T, Punnapayak H, Zimmermann W (2007) Comparison of the hydrolysis of polyethylene terephthalate fibers by a hydrolase from Fusarium oxysporum LCH I and Fusarium solani f. sp. pisi. Biotechnol J 2(3):361-364. doi:10.1002/ biot.200600095

Oeser T, Wei R, Baumgarten T, Billig S, Follner C, Zimmermann W (2010) High level expression of a hydrophobic poly(ethylene terephthalate)-hydrolyzing carboxylesterase from Thermobifida fusca KW3 in Escherichia coli BL21(DE3). J Biotechnol 146(3):100-104. doi:10.1016/j.jbiotec.2010.02.006

Ollis DL, Cheah E, Cygler M, Dijkstra B, Frolow F, Franken SM, Harel M, Remington SJ, Silman I, Schrag J, Sussman JL, Verschueren KHG, Goldman A (1992) The alpha/ beta hydrolase fold. Protein Eng 5(3):197-211. doi:10.1093/protein/5.3.197

Paszun D, Spychaj T (1997) Chemical recycling of poly(ethylene terephthalate). Ind Eng Chem Res 36(4):1373-1383. doi:10.1021/ie960563c

Ribitsch D, Herrero Acero E, Greimel K, Dellacher A, Zitzenbacher S, Marold A, Rodriguez RD, Steinkellner G, Gruber K, Schwab H, Guebitz GM (2012a) A new esterase from Thermobifida halotolerans hydrolyses polyethylene terephthalate (PET) and polylactic acid (PLA). Polymers 4(1):617-629

Ribitsch D, Herrero Acero E, Greimel K, Eiteljoerg I, Trotscha E, Freddi G, Schwab H, Guebitz GM (2012b) Characterization of a new cutinase from Thermobifida alba for PET-surface hydrolysis. Biocatal Biotransform 30(1):2-9. doi:10.3109/ 10242422.2012.644435

Ronkvist ÃM, Xie W, Lu W, Gross RA (2009) Cutinase-catalyzed hydrolysis of poly (ethylene terephthalate). Macromolecules 42(14):5128-5138. doi:10.1021/ ma9005318

Simossis VA, Heringa J (2005) PRALINE: a multiple sequence alignment toolbox that integrates homology-extended and secondary structure information. Nucleic Acids Res 33(Web Server issue):W289-W294. doi:10.1093/nar/gki390

Sulaiman S, Yamato S, Kanaya E, Kim JJ, Koga Y, Takano K, Kanaya S (2012) Isolation of a novel cutinase homolog with polyethylene terephthalatedegrading activity from leaf-branch compost by using a metagenomic approach. Appl Environ Microbiol 78(5):1556-1562. doi:10.1128/AEM.06725-11

Sztajer H, Lunsdorf H, Erdmann H, Menge U, Schmid R (1992) Purification and properties of lipase from Penicillium simplicissimum. Biochim Biophys Acta 1124(3):253-261

Thumarat U, Nakamura R, Kawabata T, Suzuki H, Kawai F (2012) Biochemical and genetic analysis of a cutinase-type polyesterase from a thermophilic Thermobifida alba AHK119. Appl Microbiol Biotechnol 95(2):419-430. doi:10.1007/s00253-011-3781-6

Wei R (2011) Hydrolysis of polyethylene terephthalate by cutinases from Thermobifida fusca KW3. PhD Thesis. Universität Leipzig, Leipzig

Wei R, Oeser T, Barth M, Weigl N, Lübs A, Schulz-Siegmund M, Hacker M, Zimmermann W (2013) Turbidimetric analysis of the enzymatic hydrolysis of polyethylene terephthalate nanoparticles. J Mol Catal B-Enzym. doi:10.1016/j. molcatb.2013.08.010
Zhang Z, Wang Y, Ruan J (1998) Reclassification of Thermomonospora and Microtetraspora. Int J Syst Bacteriol 48(2):411-422. doi:10.1099/00207713-48-2-411

Zimmermann W, Billig S (2011) Enzymes for the biofunctionalization of poly (ethylene terephthalate). In: Nyanhongo GS, Steiner W, Guebitz G (ed) Biofunctionalization of polymers and their applications. Adv Biochem Eng/ Biotechnol, vol 125. Springer, Berlin/Heidelberg, pp 97-120. doi:10.1007/ 10_2010_87

\section{doi:10.1186/s13568-014-0044-9}

Cite this article as: Wei et al:: Functional characterization and structural modeling of synthetic polyester-degrading hydrolases from Thermomonospora curvata. AMB Express 2014 4:44

\section{Submit your manuscript to a SpringerOpen ${ }^{\odot}$ journal and benefit from:}

- Convenient online submission

- Rigorous peer review

- Immediate publication on acceptance

- Open access: articles freely available online

- High visibility within the field

- Retaining the copyright to your article

Submit your next manuscript at $>$ springeropen.com 